\title{
Clinicopathological Correlation in Skin Epithelial Tumours in Tertiary Care Center in Central India
}

\author{
N. Khan ${ }^{1}$, P. Tandon ${ }^{2}$ \\ ${ }^{1}$ Department of Pathology, Gandhi Medical College, Bhopal, Madhya Pradesh, India. \\ ${ }^{2}$ Department of Pathology, Gandhi Medical College, Bhopal, Madhya Pradesh, India.
}

\section{ABSTRACT}

\section{BACKGROUND}

Late detection and diagnosis of skin epithelial tumours is primarily related to increased morbidity and mortality. Understanding the risk factors and patterns of malignancy can help early identification and prompt treatment of patients with skin tumours. In the current study, we aimed to study the pattern of skin epithelial lesions in tertiary care centre in central India and study its association with various risk factors.

\section{METHODS}

The skin epithelial lesion biopsies received in the Department of Pathology, GMC, Bhopal for histopathological evaluation during the duration of one and a half years were included in the study. Data was analysed by appropriate statistical tests using SPSS software.

\section{RESULTS}

We found that out of the 64 cases, majority of the patients belonged to the age group of 31-40 and 51-60 years with incidence being quite less among younger age groups with male preponderance. The mean age of presentation of malignant cases was significantly higher (53.2 years) than the age of presentation of benign cases (34 years). The difference was statistically significant $(p=0.003)$. Majority of the tumours occurred in the head and neck region followed by the lower limbs. The ratio of malignant cases to benign cases was 4.3:1. Among the benign lesions, Naevus and Squamous Papilloma formed bulk of the cases. Squamous Cell Carcinoma (SCC) constituted for $73 \%$ of the malignant cases. Basal cell carcinoma and malignant melanoma cases had a lower association with sun exposure as compared to SCC. Most of the cases of SCC occurred at the previous burn site.

\section{CONCLUSIONS}

We found out a wide spectrum of distribution of cases in all age groups with a greater occurrence of lesions in males than females owing to a greater extent of sun exposure in them. The study very well explained the relationship between sun exposure and SCC cases. We studied that many patients developed SCC on a site which had a burn scar. Periodic follow up of burn scar patients and non-healing lesions on head, neck and lower limb area is recommended.

\section{KEY WORDS}

Squamous Cell Carcinoma, Basal Cell Carcinoma, Malignant Melanoma, Sun Exposure, Burn Scar
Corresponding Author:

Dr. Puneet Tandon,

E-1/179, Arera Colony,

Bhopal-462016,

Madhya Pradesh, India.

E-mail: drpuneettandon@yahoo.co.in

DOI: $10.14260 / j e m d s / 2019 / 732$

Financial or Other Competing Interests: None.

How to Cite This Article:

Khan N, Tandon P. Clinicopathological correlation in skin epithelial tumours in tertiary care center in Central India. J. Evolution Med. Dent. Sci. 2019;8(45): 3374-3377, DOI:
Submission 06-09-2019,

Peer Review 24-10-2019,

Acceptance 30-10-2019,

Published 11-11-2019. 


\section{BACKGROUND}

Skin cancers are one of the most common cancers in the world with continually rising incidence and mortality rates. ${ }^{1}$ Keratoacanthoma (KA) and well-differentiated SCC are two cutaneous neoplasms that most often occur in sun-exposed sites of light-skinned persons. ${ }^{1}$ Malignant melanoma (MM) is the sixth most commonly diagnosed cancer in the USA in both genders. $^{2}$ Early diagnosis is particularly important for the survival of the patient, since malignant skin lesion can be cured successfully, if detected early. Understanding the risk factors and patterns of lesions can help early identification and prompt treatment of patients with oral cancers. Exposure to sunlight, particularly ultraviolet radiations, is clearly related to risk of development of skin cancer, including both melanoma and non-melanoma skin cancers..$^{2,3}$ Other risks include prior scars, chronic wounds, actinic keratosis, lighter skin, Bowen's disease, arsenic exposure, radiation therapy, poor immune system function, previous basal cell carcinoma, and HPV Infection. ${ }^{4}$ Epidemiologic data suggest that gender and genetics may influence the distribution of melanoma on the body surface and histopathologic characteristics of the lesion. ${ }^{5}$ The mechanisms responsible for initiating or limiting the tumours including skin types are of great importance. In the current study, we aimed to study the pattern of skin cancers in a tertiary care center located in Bhopal region in central India and study its association with various risk factors.

\section{METHODS}

This study was a cross-sectional observational study conducted in the Department of Pathology, Gandhi Medical College and Hamidia Hospital Bhopal, Madhya Pradesh from March 2017 to June 2018. All the skin biopsy tissues (For neoplastic lesions) received in the Department of Pathology, GMC, Bhopal for histopathological evaluation during the study duration were included in the study. Biopsies with tissue insufficient for histopathological evaluation and autolyzed samples were excluded from the study. The study was approved by institutional Ethics Committee of Gandhi Medical College, Bhopal (M. P). History was taken through requisition forms received in department of pathology, interview and case files. Biopsy samples were processed for H\&E staining. Sections were fixed in $10 \%$ formalin overnight at room temperature, processed and embedded in paraffin wax. Four $\mu \mathrm{m}$ sections were cut, deparaffinized and stained with $\mathrm{H} \& \mathrm{E}$ stains.

The data was analysed using appropriate statistical tests using software SPSS. Comparison of the qualitative variables between groups was done using the chi-square test and for quantitative data student $\mathrm{t}$ test was applied. $\mathrm{P}$ value was considered significant if $\mathrm{p}<0.05$, and highly significant if $\mathrm{p}<0.01$.

\section{RESULTS}

During the study period of one and a half years, a total of 64 cases of various skin epithelial tumours were extensively studied. The cases were histologically divided into Benign and Malignant categories. The various lesions were Squamous Cell Carcinoma (SCC), Basal Cell Carcinoma (BCC), Malignant Melanoma (MM), Squamous Papilloma (SP), Keratoacanthoma (KA) and Naevus (N).

We found out that out of the 64 cases, majority of the patients belonged to the age group of 31-40 and 51-60 years, followed by 61-70 years. Incidence was quite less among younger age groups. Mean Age of the cases was 50.2 years.

\begin{tabular}{|c|c|c|}
\hline Benign & Age Group & Malignant \\
\hline 1 & $0-10$ years & 0 \\
\hline 3 & $11-20$ years & 1 \\
\hline 1 & $21-30$ years & 3 \\
\hline 1 & $31-40$ years & 12 \\
\hline 2 & $41-50$ years & 7 \\
\hline 3 & $51-60$ years & 10 \\
\hline 1 & $61-70$ years & 11 \\
\hline 0 & $71-80$ years & 7 \\
\hline 0 & $81-90$ years & 1 \\
\hline 12 & Total & $\mathbf{5 2}$ \\
\hline \multicolumn{2}{|c|}{} \\
\hline \multicolumn{2}{|c|}{}
\end{tabular}

The mean age of presentation of benign cases was 34 years and mean age of presentation of malignant cases was 53.2 years. The age of presentation of benign lesions as compared to malignant lesions is significantly low. The age of presentation of malignant cases was significantly higher (Mean 53.2 years) than the age of presentation of benign cases (34 years) (Table 1). The difference was statistically significant (p-value 0.003).

Out of 64 cases, there were 43 male patients and 21 female patients that accounted for $67 \%$ and $33 \%$ respectively which was found out to be significant. Males have a higher exposure to sun owing to their occupation, and hence outweigh the number. However, when malignant cases were compared between the males and females, the difference was not statistically significant.

Out of the 64 cases, $61 \%$ belonged to the urban population whereas $39 \%$ of the cases belonged to the rural population. Of all the cases, $47 \%$ belonged to a low socioeconomic status whereas $53 \%$ belonged to a high socioeconomic status.

Majority of the tumours occurred in the head and neck region followed by the lower limbs. We found out that BCC and SCC occurred more in the head and neck region. Sites exposed more to sun had more chances of developing mutations. When the proportion of malignant tumours occurring in the head and neck region (45\%) were compared with tumours occurring in the lower Limbs (19\%), the difference was found out to be significant. This meant that sun exposed of the areas of head and neck had a greater chances to develop malignancy ( $p$-value 0.004). 
The head and neck region was further sub classified as forehead, cheeks, nasal bridge, medial canthus, eye lids, around the oral orifice, auricular region and the scalp and the percentage of lesions occurring at each site was calculated (Figure 1).

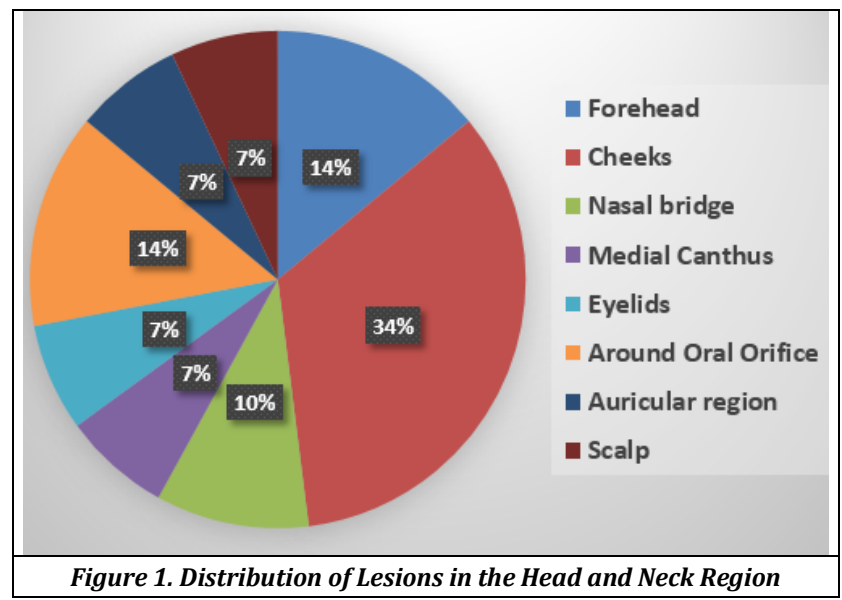

Among the head and neck region, majority of the Tumours occurred in the upper quadrants of cheeks, that is the common site for Basal Cell Carcinoma Lesions. When Basal Cell Carcinoma cases were compared with other Malignant Lesions in the head and neck region, the difference was found out to be statistically significant. So, occurrence of BCC cases in head and neck region was higher than other malignant tumours ( $p$-value 0.005). When cases of SCC were compared to other malignant Lesions occurring in the lower limbs, the difference was found out to be statistically significant. (p-value is 0.042 ) The 64 cases were further sub classified into benign and malignant categories based on the histotype. Out of the total 64 cases, 12 were found out to be benign constituting $19 \%$ of the cases whereas 52 were found out to be malignant accounting for $81 \%$ of the cases. Hence, malignancy formed the bulk of the tumours.

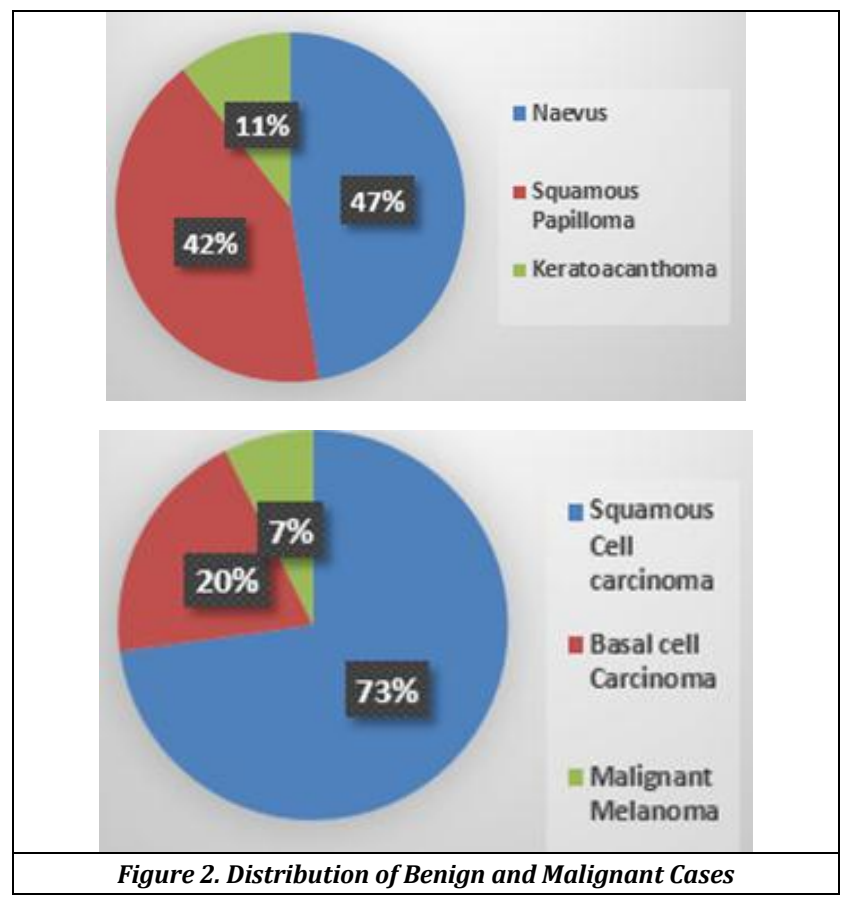

Hence, among the benign lesions, Naevus and Squamous Papilloma formed bulk of the cases. Among malignant cases, Squamous Cell Carcinoma constituted for $73 \%$ of the cases (Figure 2). The ratio of malignant cases to benign cases was 4.3:1. Out of 64 cases, $33 \%$ were associated with prolonged exposure to sun, while $67 \%$ of the cases did not give a history of a prolonged exposure. Out of the 38 cases of SCC, $47 \%$ were associated with a history of Sun exposure while $53 \%$ of the cases had no such association. BCC and MM cases had a lower association with sun exposure as compared to SCC which was statistically significant (p-value 0.029.) (Table 2).

\begin{tabular}{|c|c|c|c|c|c|c|}
\hline Risk Factors & SCC & BCC & MM & KA & NAEVUS & SP \\
\hline Burns & 10 & 0 & 0 & 0 & 0 & 0 \\
\hline Trauma & 4 & 0 & 0 & 0 & 0 & 0 \\
\hline Sun Exposure & 18 & 01 & 01 & 01 & 0 & 0 \\
\hline \\
Table 2. Association of Various Risk Factors with the Lesions \\
\hline
\end{tabular}

Most of the cases of Squamous Cell Carcinoma occurred at the previous burn site $(71 \%)$ or the site exposed to trauma (29\%) (Table 2).

\section{DISCUSSION}

The study was done to assess the pattern of skin epithelial tumours in our tertiary care center and study its association with various modifiable and non-modifiable risk factors.

A total of 64 cases presented for skin epithelial lesions in Hamidia hospital, Bhopal during the period of March 2017June 2018. The youngest patient was 10-year-old male with squamous Papilloma and the oldest patient was of a 90 -yearold female patient presenting as BCC. Hence, we came across a wide spectrum of age distribution of skin lesions. Mean age of presentation was 50.2 years. Male to female ratio was 2:1. This was comparable with other studies with a similar mean age of presentation and male predominance owing to greater sun exposure in men.

Various tumours were distributed throughout the body especially in the sun exposed skin. A majority of tumours occurred in the head and neck region accounting for $45 \%$ of all cases followed by lower limbs which constituted for $22 \%$ of the cases. A bulk of cases in the head and neck region, especially BCC and SCC correlated well with the other studies Koseoglu et al. (2009) ${ }^{6}$ In lieu with other studies Changhyum et al (2014), ${ }^{7}$ we found out that most of the cases of SCC occurred in the lower limbs (10 out of 38 cases). Hence, an early suspicion about the lesion can be made out depending upon the site of presentation. This can be helpful in early detection of the malignancy and better patient management.

The cases were segregated into benign and malignant categories. SCC, BCC and MM were the major cases diagnosed in the category of malignant tumours whereas SP, KA and Naevus formed the benign category of skin tumours. SCC formed the major bulk of tumours. Various risk factors are associated with skin tumours. Prolonged sun exposure is the commonest. We found out that $47 \%$ cases of SCC were associated with a history of prolonged sun exposure.Sunlight is a carcinogen to which everyone is exposed.

Its UV component is the major epidemiologic risk factor for squamous cell carcinoma of the skin. Of the multiple steps in tumor progression, those that are sunlight-related would 
be revealed if they contained mutations specific to UV. A study by Brash et al (1991) concluded that 14/24 (58\%) of invasive SCC of the skin contain mutations in the p53 tumor suppressor gene, each altering the amino acid sequence. UV is also implicated by a UV-like occurrence of mutations exclusively at dipyrimidine sites, including a high frequency of C----T substitutions. Lannacone et al. (2014) found a history of blistering sunburn associated with both BCC and SCC. Additionally, having a job in the sun for $\geq 3$ months for 10 years or longer (a measure of continuous sunlight exposure) was also associated with both BCC and SCC in the study population. This also suggests that sunlight exposure at a younger age is important. Advising a reduction in sunlight exposure along with sunscreen application should be done. Although squamous cell carcinoma (SCC) is the most common burn scar neoplasm, other neoplasms have also been reported. Kowal Vern A et al (2005) studied that the mean age at tumor diagnosis was 50 years, the mean age at the time of burn injury was 20 years, the mean latency interval was 31 years. Gul U et al (2006) in the case study found out that the number of SCC developing on burn scars among the 570 cases was 36 . The average latent period between burn and the development of carcinoma was found to be 32.22 years. Hence, these studies further support our explanation.

Overall, we found a wide spectrum of distribution of cases in all age groups with a greater occurrence of lesions in males than females owing to a greater extent of sun exposure in them. We observed that many patients developed SCC on a site which had a burn scar. Hence, aim should be at following post burn patients periodically. Additionally, given the head, neck and lower limb being the most common sites of malignant lesions, any lesion at these sites should be regularly followed up.

\section{CONCLUSIONS}

There is a wide distribution of cases in all age groups with a greater incidence of lesions in males owing to a greater sun exposure due to working outdoors. The study very well explained the relationship between sun exposure and SCC cases. Many patients developed SCC on a site which had a burn scar. Periodic follow up of burn scar patients and nonhealing lesions on head, neck and lower limb area can help in diagnosing such cases earlier.

\section{ACKNOWLEDGEMENT}

This article is an extract of the research project titled 'Immuno histochemical evaluation of p53, ki67 and bel-2 in skin epithelial tumours'.

\section{REFERENCES}

[1] American Joint Committee on Cancer. Cutaneous squamous cell carcinoma and other cutaneous carcinomas. AJCC Cancer Staging manual. $8^{\text {th }}$ edn. New York, NY: Springer 2017: p. 301-14.

[2] Garbe C, Leiter U. Melanoma epidemiology and trends. Clin Dermatol 2009;27(1):3-9.

[3] Curiel-Lewandrowsky C, Williams CM, Swindells KJ, et al. Use of in vivo confocal microscopy in malignant melanoma: an aid in diagnosis and assessment of surgical and non-surgical therapeutic approaches. Arch Dermatol 2004;140(9):1127-32.

[4] Ally MS, Tang JY, Arron ST. Cutaneous human papilloma virus infection and basal cell carcinoma of the skin. J Invest Dermatol 2013;133(6):1456-8.

[5] Gandhi SA, Kampp J. Skin cancer epidemiology, detection and management. The Medical Clinics of North America 2015;99(6):1323-35.

[6] Koseoglu RD, Sezer E, Eyibilen A, et al. Expressions of p53, cyclin D1 and histopathological features in basal cell carcinomas. J Cutan Pathol 2009;36(9):958-65.

[7] Changhyun K, Christine JK, David JL. Cutaneous squamous cell carcinomas of the lower extremity: a distinct subset of squamous cell carcinomas. Journal of The American Academy of Dermatologym 2014;70(1):70-4. 